\title{
TWO REDUCTIONS OF THE POINCARÉ CONJECTURE
}

\author{
BY G. A. SWARUP
}

\begin{abstract}
We study two reductions of the Poincare conjecture. The first is group theoretic and is an improvement over Papakyriakopoulos' reduction [5]. The second reduces the conjecture to a special case of it.
\end{abstract}

We first examine Papakyriakopoulos' reduction and improve it. The method also gives a new proof of a crucial theorem in his reduction.

P. 1. Conjecture. Let $G_{p}:\left\{a_{1}, b_{1}, \ldots, a_{p}, b_{p} ; \prod_{i=1}^{p}\left[a_{i}, b_{i}\right], p>1\right\}$ and let $Q_{p}=\left\{a_{1}, b_{1}, \ldots, a_{p}, b_{p} ; \Pi_{i=1}^{p}\left[a_{i}, b_{i}\right],\left[a_{1}, b_{1} \tau\right]\right\}$, where $\tau \in\left[\Phi_{p}\right.$, $\left.\Phi_{p}\right], \Phi_{p}$ being the free group generated by $a_{1}, b_{1}, \ldots, a_{p}, b_{p}$. Let $T_{p}$ be an orientable surface of genus $p$ and identity $\pi_{1}\left(T_{p}\right)$ with $G_{p}$. Then

(a) $Q_{p}$ is torsion-free.

(b) The cover of $T_{p}$ corresponding to the Kernel of the natural map $\varphi_{p}$ : $G_{p} \rightarrow Q_{p}$ is planar.

E. S. Rapaport proved [7] P.1.(a) and Papakyriakopoulos showed that P.1 implies the Poincaré conjecture [5]. He also considered the question ([5], [6]) whether P.1.(b) is group theoretic. Consider the following

P.2. Conjecture. The group $Q_{p}$ defined above is a nontrivial freeproduct.

We will show

A. Theorem. P.1 $\Rightarrow$ P.2 $\Rightarrow$ Poincaré conjecture. Moreover, P.1 is grouptheoretic.

The crucial step in the reduction [5] of Poincare conjecture to P.1 is a theorem which connects the problem of finding nontrivial simple loops in a certain normal subgroup with regular planar covers subordinate to it. This result was strengthened by Maskit in [4]; Lemmas 1 and 2 below imply his theorem. These lemmas connect the approach of Papakyriakopoulos with that of Stallings in $[8]$.

Let

$$
\{e\} \rightarrow L \stackrel{i}{\rightarrow} G \stackrel{\varphi}{\rightarrow} H \rightarrow\{e\}
$$

be an exact sequence of groups, where $G$ is the fundamental group of a closed

Received by the editors March 20, 1979.

AMS (MOS) subject classifications (1970). Primary 57A10; Secondary 20E30, 20E40, $20 \mathrm{~J} 05$.

Key words and phrases. Poincaré conjecture, planar cover, Heegaard decomposition, free product, group cohomology, structure theorem of Stallings. 
surface $T$. We are interested in finding a criterion which assests the existence of a simple loop on $T$ which represents a nontrivial element of $L$.

1. LEMMA (a) With the above notation there is a nontrivial simple loop on $T$ whose nth power for some $n$ represents an element of $L$ if there is a factorization $\varphi=q p: G \stackrel{q}{\rightarrow} Q \stackrel{p}{\rightarrow} H$ such that $H^{1}\left(Q ; \mathbf{Z}_{2} Q\right) \neq 0$.

(b) If $H$ is torsion-free, there is a nontrivial simple loop on $T$ which represents an element of $L$ if and only if $\varphi$ factors through a nontrivial free-product.

The proof of the above lemma is similar to the proof of Theorem 2 in [8]. For the first statement, we need also the structure Theorem from [9].

2. LEMMA. With the notation of Lemma 1, there is a nontrivial simple loop on $T$ whose nth power for some $n$ represents an element of $L$, if there is normal subgroup $K(\neq\{e\})$ of $G$ with $K \subset L$ and such that the cover of $T$ corresponding to $K$ is planar. The above condition is necessary [4].

Proof. Let $Q$ be the quotient of $G$ by $K$ and let $\widetilde{T}$ be the cover of $T$ corresponding to $K$. Consider the following commutative diagram

$$
\begin{aligned}
0 \rightarrow H^{1}\left(Q ; \mathbf{Z}_{2} Q\right) \rightarrow & H^{1}\left(G ; \mathbf{Z}_{2} Q\right) \\
\downarrow & \rightarrow H^{1}\left(K ; \mathbf{Z}_{2}\right) \rightarrow \\
\downarrow & H_{e}^{1}\left(\widetilde{T} ; \mathbf{Z}_{2}\right) \rightarrow H^{1}\left(\widetilde{T} ; \mathbf{Z}_{2}\right) \\
\downarrow & \downarrow \\
& H_{1}\left(\widetilde{T} ; \mathbf{Z}_{2}\right) \rightarrow H_{1}^{\infty}\left(\widetilde{T} ; \mathbf{Z}_{2}\right)
\end{aligned}
$$

The first row is exact (see [3, p. 354]). It is well known that the map in the second row is zero if and only if $\widetilde{T}$ is planar. Since $K \neq\{e\}, H_{1}\left(\widetilde{T} ; \mathbf{Z}_{2}\right) \neq 0$. Thus, if $\widetilde{T}$ is planar we have $H^{1}\left(Q ; \mathbf{Z}_{2} Q\right) \approx H^{1}\left(G ; \mathbf{Z}_{2} Q\right) \neq 0$. This completes the proof using Lemma 1.

Next, let $T$ be a two-sided orientable surface in a 3-manifold $M$. Let $M_{1}$, $M_{2}$ be the two parts into which $T$ divides $M$; if $M-T$ is connected we write $M_{2}=M_{1}$. We have two injections $i_{1}: T \rightarrow M_{1}$ and $i_{2}: T \rightarrow M_{2}$ and let $\varphi_{1}$, $\varphi_{2}$ be the induced maps in the fundamental groups. By Loop Theorem, $\varphi_{i}(T)$ is a free product of orientable surface groups and free cyclic groups and therefore $\varphi_{i}(T)$ is torsion-free. Consider $\varphi_{1} \times \varphi_{2}: \pi_{1}(T) \rightarrow \pi_{1}\left(M_{1}\right) \times \pi_{1}\left(M_{2}\right)$ and let $\varphi$ denote the induced map of $\pi_{1}(T)$ onto the image $H$ of $\varphi_{1} \times \varphi_{2}$ and let $L$ be the Kernel of $\varphi$. Then Lemma 2 implies the following.

3. TheOREM (PAPAKYRIAKopoulos, MASKit). With the above notation, there is a simple loop on $T$ which represents a nontrivial element of $L$ if and only if there is a normal subgroup $K \neq\{e\}$ of $\pi_{1}(T)$ with $K \subset L$ and such that the cover of $T$ corresponding to $K$ is planar.

That P.1 $\Rightarrow$ Poincare conjecture is proved using the above theorem when $\left(T ; M_{1}, M_{2}\right)$ is a Heegaard decomposition of a homotopy 3-sphere $M$. In this 
case $\varphi_{1} \times \varphi_{2}$ is surjective and the normal subgroup $K_{p}$ generated by the image of $\left[a_{1}, b_{1} \tau\right]$ is in the Kernel of $\varphi_{1} \times \varphi_{2}$ for some $\tau \in\left[\Phi_{p}, \Phi_{p}\right]$ (see [5]). Let $\varphi_{p}, Q_{p}$ be as in P.1. Let $\tilde{T}$ be the cover of $T$ corresponding to $K_{p}$. As in Lemma $2, \widetilde{T}$ is planar if and only if $H^{1}\left(Q_{p} ; \mathbf{Z}_{2} Q_{p}\right) \rightarrow H^{1}\left(G_{p} ; \mathbf{Z}_{2} Q_{p}\right)$ is an isomorphism. This shows that the conjecture P.1 is group-theoretic. If P.1 is true, then

$$
H^{1}\left(Q_{p} ; \mathbf{Z}_{2} Q_{p}\right) \approx H^{1}\left(G_{p} ; \mathbf{Z}_{2} Q_{p}\right) \approx H_{1}\left(K_{p} ; \mathbf{Z}_{2}\right) \neq 0
$$

Since $Q_{p}$ is torsion-free, by [9], $Q_{p}$ is a free product. Thus P.1 $\Rightarrow$ P.2. If $Q_{p}$ is a free product, then by Lemma 1 we can find a simple loop on $T$ which represents nontrivial element in $K_{p} \subset$ Kernel of $\varphi_{1} \times \varphi_{2}$ and Poincaré conjecture follows by induction. This proves Theorem A.

The above proof shows that Papakyriakopoulos' approach (via Theorem 3 above) really involves factoring $\varphi_{1} \times \varphi_{2}$ through a free product and hence it is contained in Stallings' approach [8]. However, Papakyriakopoulos has given possible candidates ( $K_{p}$ above) for finding simple loops and what we have done above seems to make his conjectures more accessible. The lemmas above can be generalized using a relative version of Stallings' theorem [10] and these can be used to prove Theorem 3 in Maskit's paper [4].

We next state another reduction of Poincare conjecture. This reduction is based on the characteristic submanifold Theory ([1] and [2]) and the results of [11]. Thurston is reported to have strong results on the existence of hyperbolic structures for the class of manifolds $T$ considered below and hence the new reduction may also be of some interest. Let $T$ denote the following class.

$M \in T$ if and only if $M$ is compact, irreducible, $X(M)=0$ and $M$ does not admit essential embeddings of the annulus or torus.

C.1. Conjecture. If $N$ is a simply connected 3-manifold obtained by adding solid tori to a $M \in T$, then $N$ is homeomorphic to $S^{\mathbf{3}}$.

C.2. Conjecture. Similar to the above, restricting $M$ to those which in addition satisfy

$\pi_{1}(M)$ is a subgroup of index $\leqslant 3$ in a real link group.

Our next reduction is

B. TheOREM. C.2 $\Rightarrow$ Poincaré conjecture.

\section{REFERENCES}

1. W. Jaco and P. Shalen, Seifert fibred pairs in 3-manifolds, Mimeographed Notes, Rice University.

2. K. Johannson, Homotopy equivalences of knot spaces, preprint, Univ. Bielefeld, 1976.

3. S. Mac Lane, Homology, Springer-Verlag, Berlin and New York, 1963.

4. B. Maskit, $A$ theorem on planar covering surfaces, with applications to 3-manifolds, Ann. of Math. (2) 81 (1965), 341-355. 
5. C. D. Papakyriakopoulos, A reduction of Poincaré conjecture to group-theoretic conjectures, Ann. of Math. (2) 77 (1963), 250-305.

6. C. D. Papakyriakopoulos, Attaching 2-dimensional cells to a complex, Ann. of

Math. (2) 78 (1963), 205-222.

7. E. S. Rapaport, Proof of a conjecture of Papakyriakopoulos, Ann. of Math. (2) 79 (1964), 506-513.

8. J. R. Stallings, How not to prove the Poincaré conjecture, Ann. of Math. Studies, vol. 60, Princeton Univ. Press, Princeton, N. J., 1966, pp. 83-88.

9. - Group theory and 3-dim. manifolds, Yale Univ. Press, 1971.

10. G. A. Swarup, Relative version of a Theorem of Stallings, J. Pure Appl. Algebra 11 (1977), 75-82.

11. Cable knots in homotopy spheres, preprint, Tata Institute, 1978.

DEPARTMENT OF MATHEMATICS, Tata Institute of Fundamental Research, Bombay 400005 , India 